\title{
The effects of pneumococcal conjugate vaccine (PCV7 and PCV13) on Turkish children with invasive pneumococcal disease: a single center experience
}

\author{
Halil Özdemir, M.D. ${ }^{a}$, Caner Ylldı, M.D. ${ }^{b}$, Selin Nar Ötgün, M.D..$^{c}$, Hatice Erkol ${ }^{b}, M . D .^{b}$, \\ Adem Karbuz, M.D. ${ }^{d}$, Bilge Aldemir Kocabaş, M.D. ${ }^{e}$, Tuğçe Tural Kara, M.D. ${ }^{a}$, \\ Ayşegül Gözalan, M.D.c, Prof., Rıza Durmaz, M.D.c, Prof., Ergin Çiftçi, M.D., Prof. ${ }^{a}$, \\ Derya Aysev, M.D. Prof.. and Erdal Ince ${ }^{a}$, M.D., Prof. ${ }^{a}$
}

a. Ankara University Medical School, Department of Pediatric Infectious Diseases, Ankara, Turkey.

b. Ankara University Medical School, Department of Pediatrics, Ankara, Turkey.

c. Public Health Agency of Turkey, Department of Microbiology Reference

Laboratories, National Molecular Microbiology Research and Application Laboratory, Ankara, Turkey.

d. Okmeydanı Training and Research Hospital, Clinics of Pediatric Infectious Diseases, İstanbul, Turkey.

e. Antalya Training and Research Hospital, Clinics of Pediatric Infectious Diseases, Antalya, Turkey.

f. Ankara University Medical School, Cebeci Hospital Microbiology Laboratory, Ankara, Turkey.

E-mail address: Halil Özdemir, M.D.: doktorhalil@gmail.com

Funding:

None.

Conflict of interest: None.

Received: 9-29-2016 Accepted: 1-16-2017

\section{ABSTRACT}

Introduction. The aim of this prospective singlecenter study was to determine the changings in incidence of invasive pneumococcal disease (IPD), serotype distribution and the antimicrobial resistance patterns of $S$. pneumoniae in children with IPD after the period ( 1 to 7 years) of vaccination with PCV7 (2008) and PCV13 (2011). Population and methods. The study was conducted on 39 Turkish children with IPD between ages 1 month and 18 years in Ankara, Turkey. Streptococcus pneumoniae was identified using standard laboratory procedures from blood, cerebrospinal fluid (CSF), pleural fluid, and other sterile body fluids and tissues. S. pneumoniae isolates were tested for resistance to penicilin and ceftriaxone using the E-test methodology. Serotypes of the isolates were determined by Quellung reaction.

Results. The overall annual incidence rate of IPD decreased significantly from $7.71(95 \% \mathrm{CI}$, 1.99-13.4) to 1.58 (95\% CI, 0.6-3.77; RRR=-79.5; $\mathrm{p}=0.006$ ) per 100000 population among $\leq 5$ years of age without underlying disease. During the overall study period, the PCV7-serotypes and PCV13-serotypes represented $27.8 \%$ and $63.8 \%$ of isolates, respectively. PCV13-serotypes made up $81.8 \%$ of cases of IPD in the pre-PCV13 era and decreased to $56 \%$ in the 4 years after PCV13. The penicillin and ceftriaxone (for meningitis) resistance rates were $48.5 \%$ and $9.1 \%$, respectively.

Conclusions. This is the first study about the changing pattern of the incidence of IPD in Turkish children after the implementation of the PCV7 and PCV13 in Turkish national vaccine schedule and a prominent decrease in incidence of IPD has seen after the implementation of PCV13.

Key words: Child; incidence; invasive pneumococcal disease; pneumococcal vaccine; Streptococcus pneumoniae.

http:/ / dx.doi.org/10.5546/ aap.2017.eng.316

To cite: Özdemir H, Yıldız C, Nar Ötgün S, et al. The effects of pneumococcal conjugate vaccine (PCV7 and PCV13) on turkish children with invasive pneumococcal disease: a single center experience. Arch Argent Pediatr 2017;115(4):316-322.

\section{INTRODUCTION}

Streptococcus pneumoniae is an important human pathogen that causes pneumonia, bacteraemia, sepsis, and meningitis, all of which result in significant morbidity and mortality worldwide especially among children under 5 years and among the elderly. ${ }^{1}$ Introduction of the 7-valent pneumococcal conjugate vaccine (PCV7) both in vaccinated children and older unvaccinated people in the United States of America (USA) and many other countries was associated with a reduction in invasive pneumococcal disease (IPD), especially on serotypes in PCV7 because of a reduction in carriage of PCV7 serotypes. However, PCV7 use modified the epidemiology of pneumococcal disease and colonization and further studies documented an increase in the rates of carriage and infections caused by nonPCV7 serotypes, thus diminishing the effect of PCV7 on overall IPD incidence. ${ }^{2,3}$

The PCV7 was introduced in Turkey in September 2005 and was included in Turkish national immunization programme in a $3+1$ schedule in November 2008 for children who were born in May 2008. In June 2011, the Ministry of Health of Turkey recommended routine vaccination with PCV13 of infants at 2, 4, 6 and 12 months old to replace PCV7, without catch-up for older children except those at high risk of IPD. Herein we aimed to determine the changings in incidence of IPD, serotype distribution and the 
antimicrobial resistance patterns of S. pneumoniae in children with IPD after the period (1 to 7 years) of vaccination with PCV7 and PCV13.

\section{POPULATION AND METHODS}

This study was conducted on children with IPD, visiting a general pediatric outpatient clinic and pediatric emergency clinic in Ankara University Faculty of Medicine, Ankara, Turkey between 2009, September $1^{\text {st }}$ and 2015, September $30^{\text {th }}$. Only immunocompetent children with IPD and S. pneumoniae isolated from blood and sterile body fluids were included in the study. The immunocompromised children and / or the children with S. pneumoniae isolated from non-sterile body fluids / tissues were excluded from the study population. The vaccination status of the children was determined as non vaccinated, fully vaccinated ( 3 or 4 doses for $<24$ months and 1 dose for $\geq 24$ months) and partially vaccinated ( 1 or 2 doses for $<24$ months) according to the Advisory Committee on Immunization Practices (ACIP) criteria. ${ }^{4}$

Isolates from blood, cerebrospinal fluid (CSF), pleural fluid, and other sterile body fluids and tissues were sent to the microbiological laboratory at Ankara University Medical School and processed in within $2 \mathrm{~h}$. The samples were inoculated in agar plates supplemented with $5 \%$ defibrinated sheep's blood and incubated overnight at $37^{\circ} \mathrm{C}$ in $5-10 \% \mathrm{CO}_{2}$ atmosphere. S. pneumoniae was identified using standard laboratory procedures, including morphology following Gram's stain, susceptibility to $5-\mu \mathrm{g}$ optochin disk and bile solubility test.

S. pneumoniae isolates were tested for resistance to penicilin and ceftriaxone using the E-test methodology. S. pneumoniae ATCC 49619 was used in antimicrobial susceptibility tests. ${ }^{5}$ Breakpoints and minimal inhibitory concentrations (MICs) were interpreted according to the 2008 Clinical Laboratory Standards Institute (CLSI). ${ }^{6}$ Briefly, the meningitis criteria for penicillin were the following: susceptible, MIC $\leq 0.06 \mu \mathrm{g} / \mathrm{ml}$; highlevel resistant, MIC $\geq 0.12 \mu \mathrm{g} / \mathrm{ml}$. Non meningitis criteria (parenteral use) for penicillin were the following: susceptible, MIC $\leq 2 \mu \mathrm{g} / \mathrm{ml}$; low-level resistant, MIC 2-4 $\mu \mathrm{g} / \mathrm{ml}$; high-level resistant, $\mathrm{MIC} \geq 8 \mu \mathrm{g} / \mathrm{ml}$; and the non-meningitis criteria (oral use) for penicillin were the following: susceptible, MIC $\leq 0.06 \mu \mathrm{g} / \mathrm{ml}$; low-level resistant, MIC 0.12-1.0 $\mu \mathrm{g} / \mathrm{ml}$; high-level resistant, MIC $\geq 2$ $\mu \mathrm{g} / \mathrm{ml}$. The meningitis criteria for ceftriaxone were the following: susceptible, $\mathrm{MIC} \leq 0.5 \mu \mathrm{g} /$ $\mathrm{ml}$; low-level resistant, $\mathrm{MIC}=1.0 \mu \mathrm{g} / \mathrm{ml}$; highlevel resistant, $\mathrm{MIC} \geq 2 \mu \mathrm{g} / \mathrm{ml}$. Non-meningitis criteria for ceftriaxone were the following: susceptible, MIC $\leq 1 \mu \mathrm{g} / \mathrm{ml}$; low-level resistant, $\mathrm{MIC}=2 \mu \mathrm{g} / \mathrm{ml}$; high-level resistant,MIC $\geq 4 \mu \mathrm{g} /$ $\mathrm{ml}$. Isolates with a MIC value higher than the susceptibility breakpoint were characterized as "non-susceptible,"i.e., low-level and high-level resistant strains. Of the isolates included in the study, $33(84.6 \%)$ were tested for antimicrobial susceptibility.

After the antibiotic susceptibility tests, the isolates were sent to Public Health Agency of Turkey, Department of Microbiology Reference Laboratories, National Molecular Microbiology Research and Application Laboratory in a coal Amies transport medium and the isolates were stored frozen in glycerol citrate at $-80^{\circ} \mathrm{C}$. Serotypes of the isolates were determined by Quellung reaction with antisera obtained from the Statens Serum Institut (Copenhagen, Denmark). Of the isolates included in the study, $36(92.3 \%)$ were serotyped.

Informed consent was obtained from the parents and the study was approved by the Ethical Committee of Ankara University Faculty of Medicine. All statistical tests were performed using the SPSS statistical package. We calculated the incidence (100 000/ outpatient admission) of IPD by using the ratio of the number of children with IPD and the number of children visiting general pediatric outpatient clinic and pediatric emergency clinic of our hospital in the same periods. Additionally, only $\leq 5$ years of age without underlying disease were taken into account for the incidence calculation. Change in incidence rate between the first year and last year study periods were assessed by calculating by absolute risks reduction and relative risks reduction (ARR and RRR).Differences were considered significant if the $p$-value was $<0.05$.

\section{RESULTS}

In this study between 2009, October $1^{\text {st }}$ and 2015 , September $30^{\text {th }} 39$ children with IPD were identified in Ankara University Children Hospital. The male/female ratio was 1.29. The median age of them were 32 months ( 1 month-18 years) and there were 29 children $(74.4 \%)$ aged under 5 years. The final diagnosis of the patients were as bacteraemia (19/39), meningitis (8/39), pneumonia $(6 / 39)$, mastoiditis and subperosteal abscess $(2 / 39)$, peritonitis $(2 / 39)$, periorbital cellulitis (1/39), and pyomyositis (1/39). Most 
of S. pneumoniae isolates $(69.2 \%)$ were collected from blood. Of the children included in the study, $19(48.7 \%)$ vaccinated $(9 / 19$ with PCV7 and 10/ 20 with PCV13) and the remaining 20 (51.3\%) children were in the partially vaccinated and non- vaccinated group. The clinical characteristics of the children with IPD and serotype distribution and antimicrobial resistance patterns of the isolates are shown in Table 1.

According to the MIC values of the isolates

TABLE 1. Clinical characteristics of the children with IPD serotype distribution and antimicrobial resistance patterns of the isolates

\begin{tabular}{|c|c|c|c|c|c|c|c|c|c|}
\hline Period & $\begin{array}{c}\text { Age } \\
\text { (month) }\end{array}$ & Gender & Diagnosis & $\begin{array}{l}\text { Site of } \\
\text { isolate }\end{array}$ & $\begin{array}{l}\text { Vaccine } \\
\text { status }\end{array}$ & $\begin{array}{l}\text { Underlying } \\
\text { disease }\end{array}$ & $\begin{array}{c}\text { Penicillin } \\
\text { susceptibility } \\
\text { (MIC) }\end{array}$ & $\begin{array}{c}\text { Ceftriaxone } \\
\text { susceptibility } \\
\text { (MIC) }\end{array}$ & Serotype \\
\hline \multirow[t]{12}{*}{ PCV7 } & 10 & $\mathrm{~F}$ & Bacteraemia & Blood & PCV7 & & 0.006 & 0.008 & $7 \mathrm{~F}$ \\
\hline & 34 & $\mathrm{~F}$ & Bacteraemia & Blood & NV & & 2 & 1 & $23 \mathrm{~F}$ \\
\hline & 27 & $\mathrm{M}$ & $\begin{array}{l}\text { Mastoiditis- } \\
\text { subperosteal } \\
\text { abscess }\end{array}$ & Abscess & NV & & 0.125 & 0.125 & $19 \mathrm{~F}$ \\
\hline & 38 & $\mathrm{~F}$ & Bacteraemia & Blood & NV & & 0.016 & 0.047 & $18 \mathrm{~F}$ \\
\hline & 6 & $\mathrm{M}$ & Bacteraemia & Blood & PV & & 0.19 & 0.19 & 14 \\
\hline & 15 & $\mathrm{~F}$ & Meningitis & CSF-Blood & PCV7 & & 0.016 & 0.002 & 10 \\
\hline & 40 & $\mathrm{~F}$ & Meningitis & CSF-Blood & NV & & 0.19 & 0.19 & $6 \mathrm{~A}$ \\
\hline & 110 & $\mathrm{M}$ & Meningitis & CSF & NV & & 1 & 0.50 & $23 \mathrm{~F}$ \\
\hline & 4 & $\mathrm{~F}$ & Bacteraemia & Blood & PV & & 0.016 & 0.008 & $7 F$ \\
\hline & 93 & M & Pneumonia & Blood & NV & & Undefined & Undefined & Undefined \\
\hline & 11 & M & Meningitis & CSF & PCV7 & & 0.64 & 0.25 & 19A \\
\hline & 3 & $\mathrm{~F}$ & Bacteraemia & Blood & PV & & 0.5 & 0.38 & $19 \mathrm{~F}$ \\
\hline \multirow[t]{27}{*}{ PCV13 } & 24 & M & Bacteraemia & Blood & PCV7 & & 0.047 & 0.023 & $15 \mathrm{~F} / \mathrm{A} / \mathrm{B} / \mathrm{C}$ \\
\hline & 9 & M & Bacteraemia & Blood & PCV7 & & Undefined & Undefined & Undefined \\
\hline & 34 & $\mathrm{~F}$ & $\begin{array}{l}\text { Periorbital } \\
\text { cellulitis }\end{array}$ & Blood & PCV7 & & 0.016 & 0.047 & $17 \mathrm{~F} / \mathrm{A}$ \\
\hline & 33 & M & Bacteraemia & Blood & PCV7 & & 0.190 & 0.190 & $19 \mathrm{~F}$ \\
\hline & 13 & $\mathrm{M}$ & Pneumonia & Blood & PCV7 & & $<0.016$ & 0.023 & 8 \\
\hline & 71 & M & Bacteraemia & Blood & NV & Hemophilia A & Undefined & Undefined & Undefined \\
\hline & 24 & M & Bacteraemia & Blood & PCV7 & & 0.75 & 0.25 & 35B \\
\hline & 117 & $\mathrm{M}$ & Peritonitis & Blood & NV & $\begin{array}{l}\text { Chronic liver } \\
\text { disease }\end{array}$ & 0.094 & 0.190 & 8 \\
\hline & 1 & $\mathrm{~F}$ & Bacteraemia & Blood & NV & & $<0.016$ & 0.008 & $7 \mathrm{~F}$ \\
\hline & 16 & M & Bacteraemia & Blood & PCV13 & & 1.5 & 1 & $15 \mathrm{~F} / \mathrm{A}$ \\
\hline & 2 & $\mathrm{~F}$ & Pyomyositis & Blood & PV & & $<0.016$ & 0.032 & 5 \\
\hline & 93 & $\mathrm{~F}$ & Peritonitis & $\begin{array}{l}\text { Peritoneal } \\
\text { fluid }\end{array}$ & NV & $\begin{array}{l}\text { Nephrotic } \\
\text { syndrome }\end{array}$ & 0.094 & 0.064 & $6 \mathrm{~A}$ \\
\hline & 19 & $\mathrm{~F}$ & Bacteraemia & Blood & PCV13 & & $<0.016$ & 0.008 & $33 \mathrm{~F} / \mathrm{A} / \mathrm{B} / \mathrm{C} / \mathrm{D}$ \\
\hline & 198 & M & Meningitis & CSF & NV & & Undefined & Undefined & $23 \mathrm{~F}$ \\
\hline & 8 & $\mathrm{M}$ & Bacteraemia & Blood & PCV13 & & 1.5 & 0.38 & $19 \mathrm{~F}$ \\
\hline & 216 & $\mathrm{~F}$ & Bacteraemia & Blood & PCV13 & $\begin{array}{c}\text { Thalassemia } \\
\text { major (post BMT) }\end{array}$ & 1 & 0.75 & $23 \mathrm{~F}$ \\
\hline & 68 & $\mathrm{M}$ & Pneumonia & Blood & NV & & 0.016 & 0.012 & 1 \\
\hline & 13 & $\mathrm{~F}$ & Bacteraemia & Blood & PCV13 & & 0.064 & 0.016 & 15B/C \\
\hline & 4 & M & Meningitis & CSF & PV & & Undefined & Undefined & $35 \mathrm{~A} / \mathrm{B} / \mathrm{C}$ \\
\hline & 40 & $\mathrm{~F}$ & $\begin{array}{l}\text { Mastoiditis- } \\
\text { subperosteal } \\
\text { abscess }\end{array}$ & Abscess & PCV13 & & 1.5 & 0.5 & $19 \mathrm{~F}$ \\
\hline & 120 & M & Meningitis & CSF & NV & Posttraumatic & Undefined & Undefined & $6 \mathrm{~A}$ \\
\hline & 4 & M & Bacteraemia & Blood & PV & & $<0.016$ & 0.008 & 12B \\
\hline & 32 & M & Pneumonia & Blood & PCV13 & & $<0.016$ & 0.004 & 3 \\
\hline & 129 & M & Pneumonia & Blood & NV & & $<0.016$ & 0.008 & $7 \mathrm{~F}$ \\
\hline & 39 & M & Bacteraemia & Blood & PCV13 & $\begin{array}{c}\text { Acute } \\
\text { lymphoblastic } \\
\text { leukemia }\end{array}$ & 0.064 & 0.016 & 3 \\
\hline & 36 & M & Pneumonia & Pleural fluid & PCV13 & & 0.75 & 0.5 & $19 \mathrm{~A}$ \\
\hline & 46 & $\mathrm{~F}$ & Meningitis & CSF-Blood & PCV13 & Posttraumatic & 0.047 & 0.047 & 21 \\
\hline
\end{tabular}


penicillin (for oral administration and parenteral administration for meningitis) resistance rate was $48.5 \%$ (16/33), but for parenteral administration of non-meningitis the penicillin resistance rate was $3.3 \%(1 / 33)$. The ceftriaxone resistance rates for meningitis and non-meningitis were $9.1 \%$ $(3 / 33)$ and $0 \%$, respectively.

During the overall study period, the PCV7serotypes and PCV13-serotypes represented $27.8 \%(10 / 36)$ and $63.8 \%(23 / 36)$ of isolates, respectively. PCV13-serotypes made up $81.8 \%$ of cases $(9 / 11)$ of IPD in the pre-PCV13 era and decreased to $56 \%(14 / 25)$ in the 4 years after PCV13. Similarly the percentage of PCV7serotypes in the cases of IPD decreased from $45.5 \%(5 / 11)$ to $20 \%(5 / 25)$ in the same period. In the first period (PCV7 era), none of the isolates were PCV7-serotype in vaccinated children and two of the three non-PCV7 serotypes (serotypes $7 \mathrm{~F}$ and 19A) were covered by PCV13. On the other hand, the serotype distribution of the partially vaccinated and non-vaccinated children was as follows: 5 were PCV-serotypes (serotype 19F: 2, serotype 23F: 2 and serotype 14: 1), 3 were nonPCV7 serotypes and two of them were covered by PCV13 (serotypes 6A and 7F) in the same period. In the second period (PCV13 era), one of the five isolate was PCV7-serotype (serotype 19F) and none of the non-PCV7-serotypes were covered by PCV13 in vaccinated children with PCV7. But, the number of the PCV13-serotypes were 6 in 10 vaccinated children with PCV13 (serotype 19F: 2, serotype 3: 2, serotype 23F: 1 and serotype 19A: 1 ) and 7 in 10 non-vaccinated children (serotype 7F: 2, serotype 6A: 2 , serotype $5: 1$, serotype $23 \mathrm{~F}: 1$, and serotype 1:1) in the same period.
The overall annual incidence rate of IPD decreased significantly from $7.71(95 \% \mathrm{CI}$, 1.99-13.4) to 1.58 (95\% CI, 0.6-3.77; RRR $=-79.5$; $\mathrm{p}=0.006$ ) per 100000 population among $\leq 5$ years of age without underlying disease. The decrease in incidence of IPD has become more evident after the implementation of PCV13 (Figure 1).

\section{DISCUSSION}

The vaccination of Turkish children with PCVs is ongoing successfully for 7 years. But, in our country information about the effects of PCV7 and PCV13 on children with IPD is not yet sufficient. There are a few studies about the serotype distribution and antimicrobial resistance patterns of pneumococci after the introduction of PCV7 and PCV13. However, those studies do not mention the changings on the incidence of pediatric IPD after the implementation of PCVs. Although this study involves a limited number of cases, it is very valuable for Turkey since it is going to provide the first information about the changing pattern of IPD incidence in Turkish children after the implementation of PCV7 and PCV13 vaccination. Also, we compared the antimicrobial susceptibility and serotype of the isolates with our country's and other countries' results prior to and post-introduction of the PCVs.

After introduction of PCV7 dramatic decreases were seen in the incidence of IPD among American children younger than 5 years. These declines were the result of the reduction in serotypes included in PCV7. However, IPD caused by non-PCV7 serotypes increased, with serotype 19A causing most incidences of IPD in the post-PCV vaccine era. Then, the achievements

FIGURE 1. The annual incidence of IPD per year in Ankara University Children Hospital

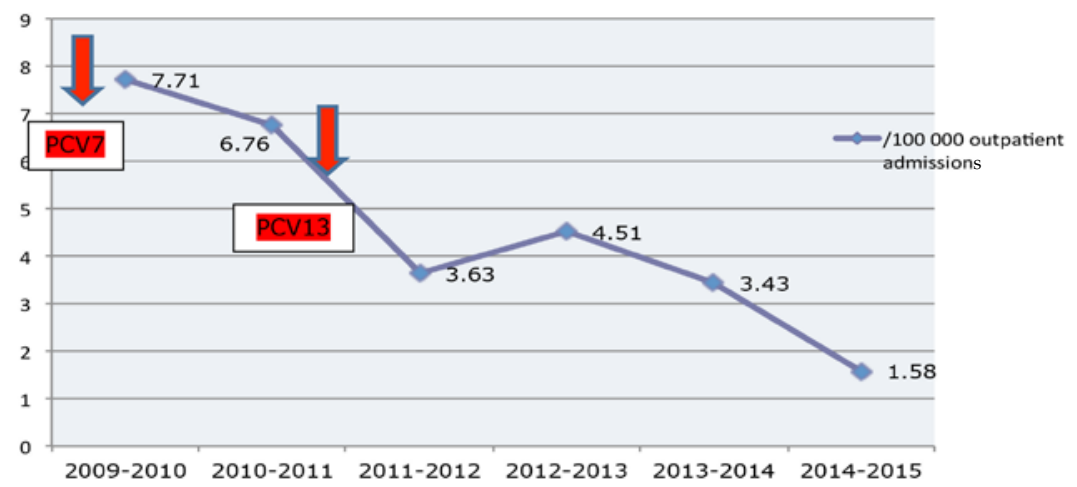


on the declining of the incidence of IPD improvement slow down. So, PCV13 replaced PCV7 for widespread use in USA. ${ }^{7,8}$ Farnham et al. reported that the incidence of IPD decreased by $69.6 \%$ from 21.0 cases per 100000 pre-PCV13 to 6.4 cases per 100000 post-PCV13 among children younger than 5 years in New York City, USA. Estimates of IPD caused by PCV13-serotypes decreased by $82.5 \%$, including a $\sim 80 \%$ reduction in serotype $19 \mathrm{~A}$ and the serotype coverage rates of the PCV13 were $72.9 \%$ and $42.1 \%$ in the pre and post-PCV13 era, respectively. ${ }^{9}$ Similar results obtained from the studies conducted on the same date intervals in Massachusetts and Alaska, USA. These studies showed a statistically significant decrease in rates of IPD and of IPD caused by PCV13-related serotypes especially in serotypes 19A, 7F and 3 after the introduction of PCV13. So that overall IPD and PCV13-serotypes IPD rates had decreased $58 \%$ and $83 \%$, respectively, in Alaska. ${ }^{8,10}$ On the other hand the number of cases of pneumococcal meningitis per year remained unchanged among US children in PCV13-era. The proportion of PCV13-serotypes decreased from $54 \%$ to $27 \%$. The most common serotypes changed as 19A, 7F and 3 to $19 \mathrm{~A}, 35 \mathrm{~B}$ and $22 \mathrm{~F}$. The penicillin susceptibility rate of the isolates was similar (75\% vs. $75 \%$ ), but the rate of the non-susceptible isolates to ceftriaxone decreased significantly from $13 \%$ to $3 \%{ }^{11}$

The incidence of IPD, serotype distribution and antimicrobial susceptibility of the pneumococci were also influenced positively by PCVs in European countries. The overall incidence of IPD across all age groups compared with the pre-PCV7 and pre-PCV13 era in England and Wales decreased by $56 \%$ and $32 \%$, respectively. But there was an evidence of increasing IPD due to non-PCV13 serotypes, particularly in children younger than 5 years in last year and these non-PCV13 serotypes were 8, 15A,15B/ $\mathrm{C}, 22 \mathrm{~F}, 23 \mathrm{~B}$ and $24 \mathrm{~F} .{ }^{3}$ The influence of PCV13 on IPD incidence was prominent in Danish children under 2 years as $71 \%$ reduction and the incidence of the 6 additional PCV13 serotypes decreased with an estimated $84 \%$ reduction. On the other hand, serotype replacement became evident and nearly $80 \%$ of causes of the IPD cases were nonPCV13 serotypes $(8,10 \mathrm{~A} / \mathrm{B}, 12 \mathrm{~F}, 15 \mathrm{~B} / \mathrm{C}, 20,22 \mathrm{~F}$, $33 \mathrm{~F}, 38,23 \mathrm{~B}, 24 \mathrm{~F})$ last year. ${ }^{12}$ Similarly, serotype switch was observed in German children. The proportion of PCV7 serotypes among isolates from IPD cases decreased from $61.8 \%$ to $5.2 \%$ and the percentage of non-PCV13 serotypes increased from $15.6 \%$ to $59.2 \%$. These non-PCV13 serotypes were $10 \mathrm{~A}, 12 \mathrm{~F}, 23 \mathrm{~B}, 24 \mathrm{~F}$ and $38 .{ }^{13}$ The important positive impact of PCV13 on the incidence of pneumococcal meningitis was examined in French children aged under 2 years. The decrease in cases due to PCV7 and to 6 additional PCV13 serotypes were $90.3 \%$ and $67 \%$, respectively. The non-PCV13 serotypes remained stable to the last year of study period, but non-PCV13 serotypes represented $67.6 \%$ of cases and mainly due to serotypes $12 \mathrm{~F}$ and $24 \mathrm{~F}$. The $39.5 \%$ of the isolates were non-susceptible to the penicillin and the $88 \%$ of them were cefotaxime/ceftriaxone-susceptible in overall of the period..$^{14,15}$

The positive effects of PCVs on the incidence of IPD were seen in two African countries, South Africa and Morocco. The rates among children younger than 2 years of age declined from 54.8 to 17.0 cases per 100000 person-years from the prePCV7, including a $89 \%$ decline in IPD caused by PCV7 serotypes in South Africa. ${ }^{16}$ Similarly, the overall annual incidence rate of IPD decreased significantly $(60.9 \%)$ among children younger than 2 years of age by implementation of the PCVs in Morocco. The overall incidence rate of IPD caused by PCV-7, PCV10-nonPCV7 and PCV13-nonPCV10 serotypes decreased by $74.1 \%$, $77.7 \%$ and $85.2 \%$, respectively. The most leading serotypes causing IPD in children younger than 2 years of age were 14,6B, 19A, 19F, 23F and 5 before vaccination. Only serotypes 6B, 14 and 1 persisted after vaccination. Also, the rates of isolates non-susceptible to penicillin decreased from $50.6 \%$ to $21.9 \% .{ }^{17}$ In Mexican children, there was a significant decrease in all PCV7 serotypes as from $59.7 \%$ to $21 \%$ and a gradual increase of the serotype 19A was detected from $7 \%$ to $39 \% .^{18}$ But, after the implementation of PCV13 there was a $75 \%$ reduction in overall IPD and no cases of serotype 19A. ${ }^{19}$

In a systematic review about the impact and effectiveness of PCV10 and PCV13 on hospitalization and mortality in children aged less than 5 years in Latin American countries (Brazil, Chile, Uruguay, Argentina, Peru and Nicaragua), the hospitalization rates of X-ray confirmed pneumonia, meningitis and IPD were declined $8.8-37.8 \%, 13.3-87.7 \%$ and $56-83.3 \%$, respectively. ${ }^{20}$ Andrade et al. showed significant impact of PCV10 on IPD for age groups targeted by vaccination in Brazil and they reported that PCV-10 serotypes decreased by $41.3 \%$ and a $44.2 \%$ reduction in IPD for children aged 2-23 months was seen. ${ }^{21}$ Similarly, one year after PCV7 introduction into 
the routine vaccination schedule of Uruguay, there was a rapid and significant reduction in rates of community acquired pneumonia (CAP), pneumococcal CAP and pneumococcal meningitis as $56 \%, 48.2 \%$ and $59 \%$, respectively. ${ }^{22}$ Also, a more significant reduction in rates of community CAP and pneumococcal CAP as $78.1 \%$ and $92.4 \%$, respectively was determined after implementation of PCV7 and PCV13 in a 9 -year period..$^{23}$

In our country, there were limited studies about the serotype distribution and antibiotic susceptibility of IPD in children. Before the implementation of PCVs, between years 2001 and 2004, Yalçın et al. reported that serotype coverage rates of PCV7, PCV10 and PCV13 were $52 \%, 74 \%$ and $81 \%$, respectively. The $39 \%$ and $14 \%$ of the isolates were non-susceptible to the penicillin and ceftriaxone, respectively. ${ }^{24}$ Then, similar results were shown in another 2 studies about the pneumococcal meningitis in children; serotype coverage rates of PCV7, PCV10 and PCV13 were $52 \%$ vs. $48.1 \%, 74 \%$ vs. $85.2 \%$ and $81 \%$ vs. $92.6 \%$, respectively. The most common serotypes were 1, 5, 6A/B, $19 \mathrm{~F}$ and $23 \mathrm{~F} .{ }^{25,26}$ The PCVs firstly show their effects on the serotype replacement of nasopharyngeal colonization and we experienced that phenomen during the 3 years following the introduction of PCV7 to the Turkish national vaccine schedule in our previous study conducted on healthy children. The rates of serotype covered by PCV7 and PCV13 were $46.2 \%$ and $62 \%$, respectively. ${ }^{27}$ The increasing of nonvaccine serotypes of nasopharyngeal colonization influence the serotype distribution of the isolates causing IPD. In this study the PCV7-serotypes and PCV13-serotypes represented $27.8 \%$ and $63.8 \%$ of isolates, respectively. PCV13-serotypes made up $81.8 \%$ of cases of IPD in the pre-PCV13 era and decreased to $56 \%$ in the 4 years after PCV13. Similarly the percentage of PCV7-serotypes in the cases of IPD decreased from $45.5 \%$ to $20 \%$ in the same period. Similarly, Ceyhan et al. showed that the potential serotype coverage ranged from $57.5 \%$ to $36.8 \%$ and from $77.4 \%$ to $60.5 \%$ for PCV7 and PCV13 in 2008-2014 (pre-PCV7 and postPCV13 era) in Turkey in children aged $\leq 5$ years, respectively. Also, they found that the percentage of non-PCV13 serotypes was 37.6 in PCV13 era and in our study the non-PCV13 serotypes rate was $44 \%$ in PCV13 era. ${ }^{28}$ Although serotype 19A was the most responsible serotype in children with IPD in USA and in European countries in post PCV7-era, as a surprising result, there was only 1 serotype 19A in our patients in post PCV7 and PCV13-era. Also, serotype 19A was rare in Turkish children with IPD as $1.8 \%$ to $5.6 \% .{ }^{24-26,28}$ On the other hand, serotype $19 \mathrm{~F}$ was identified in 3 fully vaccinated children with PCV7 or PCV13.

\section{CONCLUSIONS}

In conclusion, this is the first study of a single center about the changing pattern of the incidence of IPD in Turkish children after the implementation of the PCV7 and PCV13 in Turkish national vaccine schedule and a prominent decrease in incidence of IPD has seen after the implementation of PCV13.

\section{REFERENCES}

1. Park M, Kim HS, Shin KS, et al. Changes in the incidence of Streptococcus pneumoniae bacteremia and its serotypes over 10 years in one hospital in South Korea. Vaccine 2014;32(48):6403-7.

2. Mendes RE, Costello AJ, Jacobs MR, et al. Serotype distribution and antimicrobial susceptibility of USA Streptococcus pneumoniae isolates collected prior to and post introduction of 13-valent pneumococcal conjugate vaccine. Diagn Microbiol Infect Dis 2014;80(1):19-25.

3. Waight PA, Andrews NJ, Ladhani SN, et al. Effect of the 13-valent pneumococcal conjugate vaccine on invasive pneumococcal disease in England and Wales 4 years after its introduction: an observational cohort study. Lancet Infect Dis 2015;15(5):535-43.

4. Centers for Disease Control and Prevention (CDC), Advisory Committee on Immunization Practices. Updated recommendation from the Advisory Committee on Immunization Practices (ACIP) for use of 7-valent pneumococcal conjugate vaccine (PCV7) in children aged 24-59 months who are not completely vaccinated. MMWR Recomm Rep 2008;57(13):343-4.

5. Centers for Disease Control and Prevention (CDC). Laboratory methods for the diagnosis of meningitis caused by Neisseria meningitidis, Streptococcus pneumoniae, and Haemophilus influenzae. WHO manual. 2nd ed. Atlanta, GA: WHO Press; 2011:1-14. [Access: 17 de enero de 2017] Disponible en: https://www.cdc.gov/meningitis/labmanual/full-manual.pdf

6. Clinical and Laboratory Standards Institute. Performance standards for antimicrobial susceptibility testing; 19th informational supplement. M100-S19. Wayne, PA: Clinical and Laboratory Standards Institute; 2009.

7. Wenger JD, Zulz T, Bruden D, et al. Invasive pneumococcal disease in Alaskan children: impact of the seven-valent pneumococcal conjugate vaccine and the role of water supply. Pediatr Infect Dis J 2010;29(3):251-6.

8. Bruce MG, Singleton R, Bulkow L, et al. Impact of the 13-valent pneumococcal conjugate vaccine (pcv13) on invasive pneumococcal disease and carriage in Alaska. Vaccine 2015;33(38):4813-9.

9. Farnham AC, Zimmerman CM, Papadouka V, et al. Invasive pneumococcal disease following the introduction of 13-valent conjugate vaccine in children in New York City from 2007 to 2012. JAMA Pediatr 2015;169(7):646-52.

10. Iroh Tam PY, Madoff LC, Coombes B, et al. Invasive pneumococcal disease after implementation of 13-valent conjugate vaccine. Pediatrics 2014;134(2):210-7. 
11. Olarte L, Barson WJ, Barson RM, etal.Impact of the 13-valent pneumococcal conjugate vaccine on pneumococcal meningitis in US children. Clin Infect Dis 2015;61(5):767-75.

12. Harboe ZB, Dalby T, Weinberger DM, et al. Impact of 13 -valent pneumococcal conjugate vaccination in invasive pneumococcal disease incidence and mortality. Clin Infect Dis 2014;59(8):1066-73.

13. Van der Linden $M$, Falkenhorst G, Perniciaro $S$, et al. Effects of infant pneumococcal conjugate vaccination on serotype distribution in invasive pneumococcal disease among children and adults in Germany. PLoS One 2015;10(7):e0131494.

14. Chapoutot AG, Dessein R, Guilluy O, et al. Impact of the 13-valent pneumococcal conjugate vaccine on the incidence of pneumococcal meningitis in children. Epidemiol Infect 2016;144(3):607-11

15. Levy C, Varon E, Picard C, et al. Trends of pneumococcal meningitis in children after introduction of the 13-valent pneumococcal conjugate vaccine in France. Pediatr Infect Dis J 2014;33(12):1216-21.

16. von Gottberg A, de Gouveia L, Tempia S, et al. Effects of vaccination on invasive pneumococcal disease in South Africa. N Engl J Med 2014;371(20):1889-99.

17. Diawara I, ZeroualiK, Katfy K, etal. Invasive pneumococcal disease among children younger than 5 years of age before and after introduction of pneumococcal conjugate vaccine in Casablanca, Morocco. Int J Infect Dis 2015;40:95-101.

18. Echaniz-Aviles G, Soto-Nogueron A, Miranda-Novales G, et al. Streptococcus pneumoniae serotypes identified in Mexican children with invasive disease before and after the introduction of PCV7 (1993-2012). Arch Med Res 2015;46(2):149-53.

19. Chacon-CruzE, Rivas-Landeros RM, Volker-Soberanes ML. Early trends in invasive pneumococcal disease in children following the introduction of 13-valent pneumococcal conjugate vaccine: results from eight years of active surveillance in a Mexican hospital. Ther Adv Vaccines 2014;2(6):155-8.
20. deOliveira LH, CamachoLA, CoutinhoES, et al. Impact and effectiveness of 10 and 13-valent pneumococcal conjugate vaccines on hospitalization and mortality in children aged less than 5 years in Latin American countries: a systematic review. PLoS One 2016;11(12):e0166736.

21. Andrade AL, Minamisava R, Policena G, et al. Evaluating the impact of PCV-10 on invasive pneumococcal disease in Brazil: a time-series analysis. Hum Vaccin Immunother 2016;12(2):285-92.

22. Pírez MC, Algorta G, Cedrés A, et al. Impact of universal pneumococcal vaccination on hospitalizations for pneumonia and meningitis in children in Montevideo, Uruguay. Pediatr Infect Dis J 2011;30(8):669-74.

23. Pírez MC, Algorta G, Chamorro F, et al. Changes in hospitalizations for pneumonia after universal vaccination with pneumococcal conjugate vaccines $7 / 13$ valent and Haemophilus influenzae type $\mathrm{b}$ conjugate vaccine in a pediatric referral hospital in Uruguay. Pediatr Infect Dis J 2014;33(7):753-9.

24. Yalçın I, Gürler N, Alhan E, et al. Serotype distribution and antibiotic susceptibility of invasive Streptococcus pneumoniae disease isolates from children in Turkey, 2001-2004. Eur J Pediatr 2006;165(9):654-7.

25. Toprak D, Soysal A, Torunoğlu MA, et al. PCR-based national bacterial meningitis surveillance in Turkey: years 2006 to 2009. Pediatr Infect Dis J 2014;33(10):1087-9.

26. Ceyhan M, Yildirim I, Sheppard CL, et al. Pneumococcal serotypes causing pediatric meningitis in Turkey: application of a new technology in the investigation of cases negative by conventional culture. Eur J Clin Microbiol Infect Dis 2010;29(3):289-93.

27. Özdemir H, Çiftçi E, Durmaz R, et al. Nasopharyngeal carriage of Streptococcus pneumoniae in healthy Turkish children after the addition of PCV7 to the national vaccine schedule. Eur J Pediatr 2014;173(3):313-20.

28. CeyhanM,OzsurekciY, GürlerN, etal.Serotypedistribution of Streptococcus pneumoniae in children with invasive diseases in Turkey: 2008-2014. Hum Vaccin Immunother 2016;12(2):308-13. 